\title{
Axotomy-Induced Axonal Degeneration Is Mediated by Calcium Influx Through lon-Specific Channels
}

\author{
Edwin B. George, Jonathan D. Glass, and John W. Griffin \\ Department of Neurology, School of Medicine, Johns Hopkins University, Baltimore, Maryland 21287-6953
}

We examined the role of extracellular calcium entry, the possible involvement of axonal calcium channels, and the potential protective effect of calcium channel and calpain antagonists in axotomy-induced axonal degeneration using murine dorsal root ganglia in cell culture. We found that calcium entry is both necessary and sufficient to induce axonal degeneration after axotomy, and may be inhibited by cobalt, manganese, dihydropyridines, and bepridil. Tetrodotoxin and $\omega$-conotoxin are ineffective in preventing axonal degeneration. The activation of calpains also appears to be necessary and sufficient for axonal degeneration to proceed, and can be blocked with membrane-permeant leupeptin analogs and the oxirane aloxistatin. Although other calcium-activated events may occur, it appears that inhibition of calpain is sufficient to preserve the axon at the light microscope level, and to prevent axonal cytoskeleton degradation as detected by immunofluorescent staining.

Our results suggest that axonal degeneration after axotomy involves the following sequence of events: (1) a lagperiod after axotomy prior to the onset of axonal degeneration, (2) entry of calcium into the axon through an intact axolemma via a calcium-specific ion transport mechanism, (3) activation of calcium-dependent effector molecules such as calpains, (4) degradation of the axonal cytoskeleton. The details of the second step require further elucidation, and are of particular interest because this step is a potential target for therapies directed towards peripheral neuropathies.

[Key words: axons, axotomy, calcium, calcium channel blockers, calcium channels, calpain, cultured cells, cytoskeleton, dihydropyridines, sensory ganglia, immunohistochemistry, mice, inbred c57bl mice, nerve degeneration, nerve fibers, neurons, neuropathy, Wallerian degeneration]

Nerve fibers require continuity with the nerve cell body for their survival. This principle, implicit in Waller's observation that the distal stumps of transected nerve fibers degenerate, was one of the fundamental discoveries in nineteenth century neurobiology (Waller, 1850). The recognition 100 years later that most neuronal protcins arc synthesized in the neuronal perikaryon and carried to the axon by specialized axonal transport systems led to the attractive presumption that degeneration of the transected

\footnotetext{
Received Jan. 12, 1995; revised May 2, 1995; accepted May 9, 1995.

E.B.G. was supported in part by NIH 5 K08 NS01504.

Correspondence should be addressed to Edwin B. George, M.D., Ph.D., Pathology Building 509, 600 North Wolfe Street, Baltimore, MD $21287-6953$.

Copyright (C 1995 Society for Neuroscience 0270-6474/95/156445-08\$05.00/0
}

axon was due to its disconnection from the cell body, resulting in a form of intracellular "starvation" for necessary proteins and other materials. While this may be the case in some species (Cancalon and Elam, 1980; Cancalon, 1983a,b), reassessment of the basis for degeneration of the transected mammalian nerve fiber (Wallerian degeneration) is mandated by the recent discovery of a mutant mouse substrain, the $\mathrm{C} 57 / \mathrm{Bl} / \mathrm{Ola}$ (Ola) mouse, whose axons survive for as long as a month after transection (Lunn et al., 1989; Glass and Griffin, 1991; Brown et al., 1994; Tsao et al., 1994).

The prolonged survival of transected Ola axons demonstrates that a general depletion of materials carried by axonal transport cannot be the cause of axonal degeneration in normal axons, which degenerate within a few days of transection. Instead, it appears that a specific mechanism must be present in normal axons, but not in Ola axons, which leads to prompt axonal degeneration once continuity with the cell body is lost. Since Ola mice are otherwise normal, it appears that it is possible to prevent the normal mechanism of prompt axonal degeneration without greatly perturbing the other properties of the neuron.

The existence of an axonal degeneration mechanism that can both be triggered by certain events and be specifically blocked carries profound implications for the physiology and pathophysiology of axons. Axonal degeneration is an important step in allowing for subsequent regeneration after axon injury. Degeneration of unwanted axons and pruning of axonal arborizations is a key element during nervous system development. Axonal degeneration is a common process in a wide variety of metabolic, toxic, hereditary, and inflammatory disorders. The morphology of axonal degeneration is very similar in all these circumstances, and the existence of a specific mechanism for axonal degeneration suggests that there may be common elements in the axonal degeneration which occur in each of these cases.

The simplest model of axonal degeneration remains Wallerian degeneration produced by nerve transection. Studies in the 1970s demonstrated that severed axons maintained in EGTA degenerated more slowly than those in high-calcium media, and implicated the action of axonal calcium-dependent proteases (calpains), which were demonstrated to cleave the major axonal proteins (Schlaepfer and Bunge, 1973; Schlaepfer, 1974; Schlaepfer and Micko, 1978; Schlaepfer and Hasler, 1979; Schlaepfer and Zimmerman, 1984; Schlaepfer et al., 1985). Proteolytic activity appears to lead to granular disintegration of the axoplasm, the first morphologically detectable change in an axon undergoing axonal degeneration. The mechanism by which axonal calcium levels might increase sufficiently to trigger granular disintegration of the axon has not been established. Using murine dorsal root ganglia in cell culture, we examined the role of 
extracellular calcium entry, the possible involvement of axonal calcium channels, and the potential protective effect of calcium channel and calpain antagonists.

\section{Materials and Methods}

The typical experiment involved the following steps. Dorsal root ganglion neurons were allowed to extend axons in culture. The axons were subsequently severed near the cell body, and the distal axon was allowed to degenerate for $4 \mathrm{~d}$. The degenerating axons were subjected to various pharmacologic treatments during this period, and then the extent of axonal degeneration was determined at the end of the $4 \mathrm{~d}$ period. The degenerating axons were subsequently fixed and prepared for immunostaining.

Dorsal root ganglion cultures. The dorsal root ganglia were dissected from mouse pups during the first two days of life. Whole ganglia explants were cultured in collagen-coated $35 \mathrm{~mm}$ dishes (Falcon) using N2 medium (Dulbecco's Modified Eagle medium and Ham's F12 medium (GIBCO) mixed 1:1 and supplemented with progesterone, selenium, insulin, putrescine, glucose, 7S NGF, and penicillin-streptomycin) at $37^{\circ} \mathrm{C}$ in a $5 \% \mathrm{CO}_{2}$ environment (Bottenstein and Sato, 1979). Axons grew outwards from the ganglia, producing a neuritic halo. Ganglia were cultured for $10-15 \mathrm{~d}$ prior to experiments in order to obtain sufficient axon growth.

Materials. Media with reduced calcium concentrations were prepared from powdered depleted Dulbecco's Modified Eagle/Ham's F12 medium (Sigma) supplemented with the usual amounts of all depleted components except for $\mathrm{CaCl}_{2}$, which was added to the desired concentration of calcium, and additional $\mathrm{MgCl}_{2}$ to keep the sum of the calcium and magnesium concentrations constant. The free $\left[\mathrm{Ca}^{2+}\right]$ in reduced calcium and in EGTA containing media was determined using a calcium-specific electrode (Orion). EGTA, nifedipine, and aloxistatin were obtained from Sigma. Calpain inhibitor I, calpain inhibitor II, $\omega$-conotoxin GVIA, and bepridil were obtained from CalBiochem. The antibodies other than anti- $\beta$-tubulin (Sigma), PGP9.5 (Accurate) and anti-neurofilament (Sternberger Monoclonals) were purchased from Molecular Probes. The ionophores A23187 and ionomycin were purchased from Molecular Probes. Nimodipine was the gift of Alexander Scriabine (Miles).

Axotomy-induced axonal degeneration. Using a microscalpel, cuts were made through the neuritic halo around each ganglion and the ganglion was removed, leaving the severed axons in the culture dish. Excision of the ganglia prevents axons from regenerating into the region occupied by the degenerating axons. Within $30 \mathrm{~min}$ after axotomy the culture medium was exchanged with media containing the desired pharmacologic agents, or in some cases with media with a reduced calcium concentration.

Axonal degeneration occurred over a period of several days after axotomy. In control cultures, the severed axons appeared normal for 12-24 hr after axotomy, then developed focal swellings or beads. In most cases, the axons completely degenerated by $48-60 \mathrm{hr}$, leaving little or no axonal debris. Some transected axons took as long as $72-84 \mathrm{hr}$ to completely degenerate. To allow for complete degeneration to occur, the effects of various treatments were assessed by comparison to control cultures at $96 \mathrm{hr}$ after axotomy.

Data analysis. Four days after axotomy, the cultures were examined under a phase-contrast microscope (Olympus IMT-2). Each ganglion excision (axotomy) site was evaluated for the presence of surviving axons. Axons with a normal appearance and axons with a beaded appearance were scored as having survived. Discontinuous fragments of what appeared to have been an axon were considered to be debris, and the axon was scored as degenerated. Each ganglion excision site was scored as either having surviving axons or not. For each experiment, the fraction of ganglion sites with surviving axons was calculated and the binomial standard deviation determined.

This approach gives an "all or nothing" score to each ganglion explant. In doing so it eliminates most observer bias, by asking for each ganglion explant "Are there any remaining axons which meet the simple morphologic criteria for preservation?" The abundance of the preserved axons (which varies with the abundance of axons prior to axotomy) and the vitality of the preserved axons need not be judged by the observer, although these subjective impressions do seem to correlate with the fraction of ganglion sites which have surviving axons. The measure is relatively insensitive to degeneration of some but not all axons: if all ganglia lose $50 \%$ of their axons, we would still score $100 \%$ survival. There is, however, considerable ganglion-to-ganglion variation

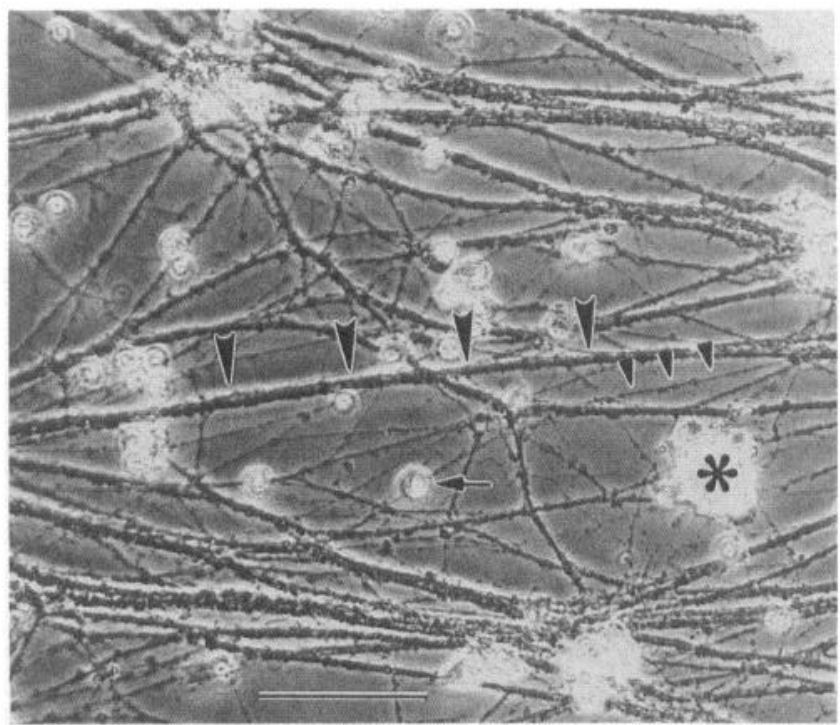

Figure 1. Axons in a murine dorsal root ganglia culture $4 \mathrm{~d}$ after axotomy, kept in media with EGTA. The culture medium was exchanged within $30 \mathrm{~min}$ after axotomy with media containing $2 \mathrm{~mm}$ EGTA. Free $\left[\mathrm{Ca}^{2+}\right]$ in the media was $<1.0 \mu \mathrm{M}$ as determined by calcium-specific electrode. The axons develop some beading, which is clearly seen along individual axons and small fascicles (small arrowheads). The beading of the individual axons creates a "grainy" appearance in the large fascicles (large arrowheads). Low calcium concentrations cause non-neuronal cells to lose their adherence to the substratum, and develop a rounded, swollen appearance and increased brightness in these phase-contrast micrographs. One such cell is designated by the small arrow, while a cluster of cells is marked with an asterisk. Scale bar, $25 \mu \mathrm{m}$.

in the abundance and vigor of the axon outgrowth under the best of conditions, and conditions which lead to a noticeable reduction in axon survival among the most luxuriant ganglia generally produce a greater percentage of ganglia with no surviving axons.

Immunohistochemistry. Cultures to be immunostained were fixed with $4 \%$ formaldehyde in the culture dish for one hour. The cultures were washed with phosphate buffer, then exposed to primary antibody for at least $1 \mathrm{hr}$, washed again, and incubated with FITC-conjugated (1: $25)$ or Texas red-conjugated $(1: 100)$ secondary antibody for at least 1 hr. Primary antibodies used included anti- $\beta$-tubulin (1:100), anti-neurofilament (1:1000), and anti-PGP9.5 (1:500). BODIPY-phalloidin (1: 100) was used to stain cultures for actin. Cultures were fixed as described and then exposed to BODIPY-phalloidin for $1 \mathrm{hr}$. The immunostained cultures were observed and photographed under phase-contrast and epifluorescence using the Olympus IMT-2 microscope.

\section{Results}

External calcium is required for axonal degeneration

When the neurites elaborated by cultured murine dorsal root ganglia are transected (axotomy), the severed axons appear normal for 12-24 hr, then develop focal swellings or beads. In most cases, the axons completely degenerate by $48-60 \mathrm{hr}$, leaving little or no axonal debris. Some transected axons take as long as $72-84 \mathrm{hr}$ to completely degenerate. When extracellular calcium levels are reduced by adding EGTA to the culture media after axotomy, the axons develop a beaded appearance but fail to degenerate within a week after axotomy (Fig. 1). When additional calcium is added to maintain normal free calcium concentrations in the presence of EGTA, axonal degeneration proceeds.

Axonal degeneration is also delayed when axotomized axons are exposed to media with a reduced calcium content. To reliably 


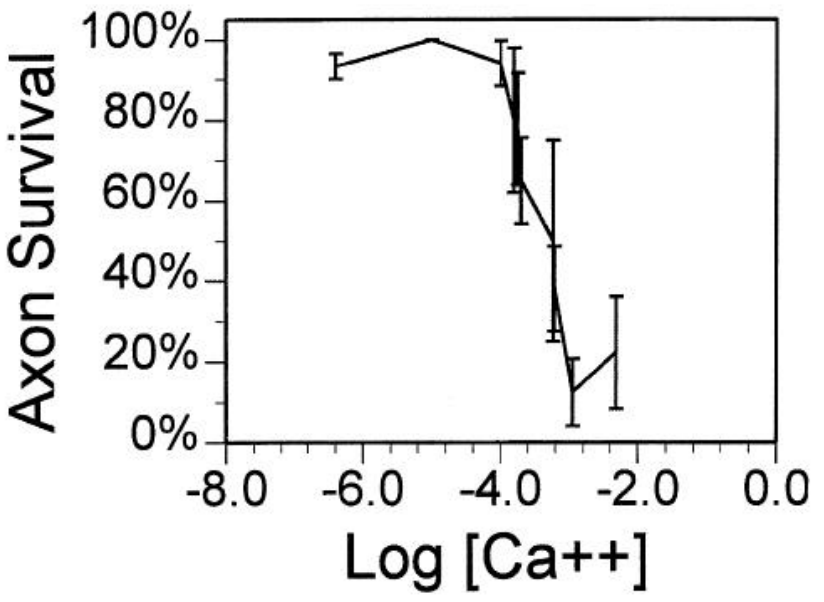

Figure 2. The dependence of axonal degeneration on external calcium concentration. The y-axis represents the percent of cultured ganglia showing axonal preservation at $4 \mathrm{~d}$ after axotomy. The $\mathrm{x}$-axis shows the $\log \left[\mathrm{Ca}^{2+}\right]$. Data points for calcium concentration greater than $1 \mathrm{e}-4$ $(100 \mu \mathrm{M})$ were obtained without EGTA. The actual concentration of free calcium in each culture was determined using an ion specific electrode. Each point represents at least 10 experiments. Error bars are \pm 1 binomial SD.

attain calcium concentrations less than $100 \mu \mathrm{M}$, EGTA must be added to the culture media to buffer the calcium concentration from variations due to the small amounts of calcium in the water and culture reagents. For concentrations above $100 \mu \mathrm{M}$, media without added calcium is prepared and the required amount of calcium is added. The actual concentration of free calcium in each culture is determined using an ion specific electrode. When extracellular calcium is reduced below $200 \mu \mathrm{M}$, the axotomized axons fail to degenerate for at least $4 \mathrm{~d}$ (Fig. 2).

\section{Calcium entry is sufficient to produce axonal degeneration}

An increase in the intra-axonal calcium concentration is capable of producing axonal degeneration which appears identical to the degeneration produced by axotomy. Elevations of intra-axonal calcium can be produced with calcium ionophores. Both A23187 and ionomycin produce degeneration of uncut axons when added to dorsal root ganglia cultures (Fig. 3). Ionophores are added to both uncut and axotomized cultures at $20 \mathrm{~nm}$. Axonal degeneration is evident by light microscopy within $30 \mathrm{~min}$ after ionophore addition, and axons are completely disrupted by $1 \mathrm{hr}$, although identifiable axonal debris persists. Rapid degeneration of cut axons is produced when the ionophore is added within $24 \mathrm{hr}$ of axotomy, indicating that the neuronal cell body is not involved in the axonal response to ionophores. Simultaneous addition of EGTA prevents the axonal degeneration induced by ionophores, confirming that it is a calcium dependent process.

\section{Calcium channel blockers prevent axonal degeneration}

Cobalt and manganese are non-specific calcium channel inhibitors: these metal ions prevent calcium entry by their ability to enter and be bound within calcium-specific channels. Both cobalt and manganese are effective in delaying axonal degeneration after axotomy. Figure 4 shows the dose-response relation for preservation of axotomized axons by cobalt and manganese. Cobalt has a lower $\mathrm{EC}_{50}$ than is seen with manganese. Addition of the calcium ionophore A23187 in the presence of $1 \mathrm{~mm}$ cobalt results in rapid axonal degeneration, confirming that the effect
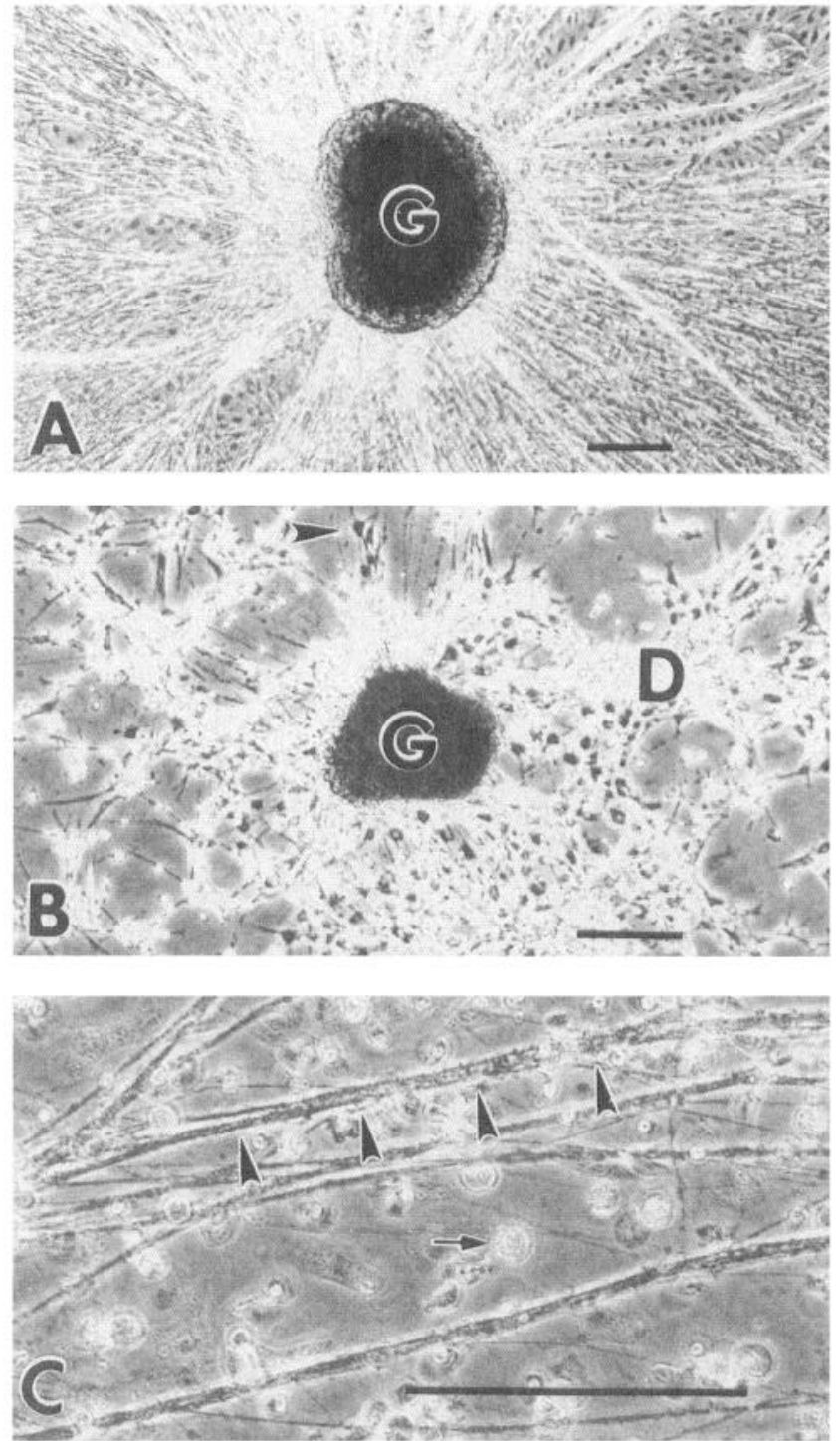

Figure 3. Axonal degeneration induced by calcium ionophore A23187. A, An untreated cultured dorsal root ganglion $(G)$ is seen in the center of this phase-contrast micrograph, with copious axons radiating outwards. Axons emerging from the ganglion above the plane of focus appear bright, but are seen as fine dark fibers once they reach the substratum and are in focus. This is well seen in the left upper quadrant of the panel. In the upper right quadrant, the nuclei of non-neuronal cells are evident in an area with few overlying axons. B, A cultured

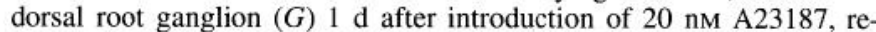
sulting in axonal degeneration. The massed debris from the degenerated axons and non-neuronal cells appears bright $(D)$, while some non-neuronal cells remain on the substratum and appear dark (arrowhead). The few remaining fine fibers are short, and most likely are Schwann cell processes, although they could be axonal fragments. The same effect is obtained with $20 \mathrm{~nm}$ ionomycin (not shown). $C$, Cultured axons $1 \mathrm{~d}$ after introduction of $20 \mathrm{nM} \mathrm{A23187}$ and 2 mM EGTA, showing preservation of the axons (compare with Fig. 1). The beading of the individual axons creates a "grainy" appearance in the large fascicles (arrow heads). Non-neuronal cells appear rounded, swollen and bright (small arrow). Scale bars, $50 \mu \mathrm{m}$.

of cobalt appears to be due to prevention of calcium entry via an ion-specific channel.

Two dihydropyridine calcium channel antagonists, nifedipine and nimodipine, delay axonal degeneration after axotomy in a dose dependent fashion (Fig. 5). The $\mathrm{EC}_{50}$ values were 9.5 and 

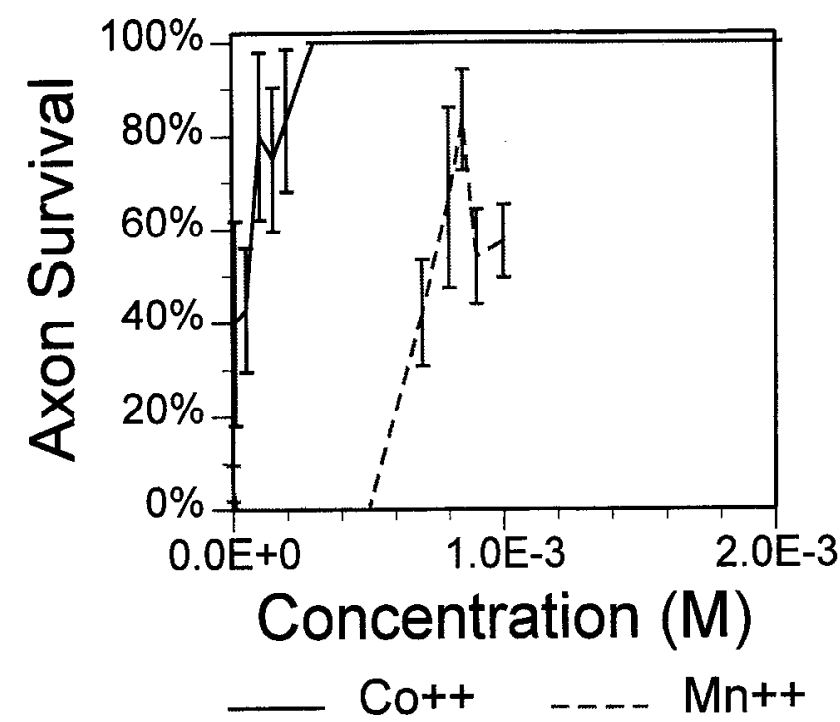

Figure 4. Cobalt and manganese inhibition of axonal degeneration. Axonal preservation at $4 \mathrm{~d}$ is plotted against $\left[\mathrm{Co}^{2+}\right]$ (solid line), and against $\left[\mathrm{Mn}^{2+}\right]$ (broken line). For each point, $n \geq 6$. Error bars are \pm biromial SD.

13.7 $\mu \mathrm{M}$, respectively. These agents are known to block highthreshold noninactivating (L-type) calcium channels. The effective inhibitory concentrations we observed are slightly higher than those reported for neuronal L-channels in electrophysiologic experiments (Kostyuk, 1992). This may reflect a requirement for a high degree of channel blockade in order to prevent calcium accumulation in the axons over the time course of our experiments.

\section{Conotoxin does not block axonal degeneration}

$\omega$-Conotoxin GVIA is a peptide toxin purified from the venom of cone snails. $\omega$-Conotoxin GVIA blocks high-threshold inactivating (N-type) calcium channels in chick dorsal root ganglion, rat sympathetic cells and PC12 cells (Kasai et al., 1987; Olivera et al., 1987; Plummer et al., 1989; Hess, 1990). $\omega$-Conotoxin GVIA does not inhibit axonal degeneration in our cultures even at very high concentrations (up to $10 \mathrm{~nm}$ ). This suggests that calcium enters primarily through channels other than $\mathrm{N}$-type calcium channels during axonal degeneration.

\section{Bepridil blocks axonal degeneration}

In addition to blocking sodium- and calcium-specific ion channels, bepridil blocks the sodium-calcium exchanger (Braun et al., 1992; Gill et al., 1992; Kostyuk, 1992). By blocking the sodium-calcium exchanger, bepridil normally should act to enhance calcium accumulation, although under certain circunstances, discussed in more detail below, the sodium-calcium exchanger may promote calcium entry, and bepridil would have the opposite effect. If the primary cause of the rise in intracellular calcium were failure to extrude or sequester intracellular calcium, and not an influx of calcium through calcium specific channels, bepridil would be expected to promote axonal degeneration. Bepridil prevents axonal degeneration at low concentrations, but the inhibition of axonal degeneration gradually decreases with increasing concentration (Fig. 6). This result is still consistent with calcium entry via ion specific channels as an important component of the increase in intra-axonal calcium, and suggests that the inhibitory effect of bepridil on the sodium-

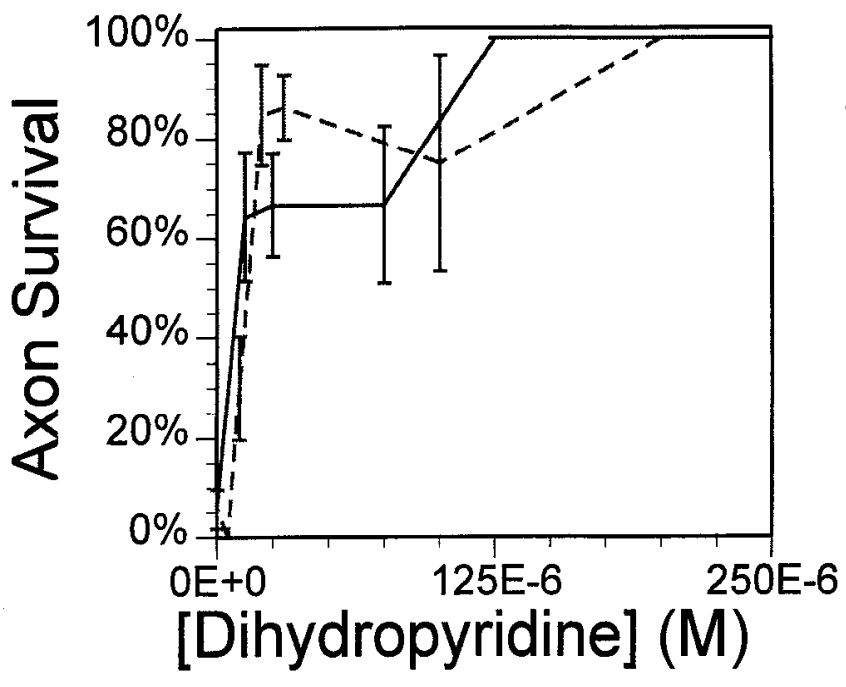

Nifedipine --.-- Nimodipine

Figure 5. Dihydropyridine inhibition of axonal degeneration. Axonal preservation at $4 \mathrm{~d}$ is plotted against nifedipine (solid line) and nimodipine (broken line). The $\mathrm{EC}_{50}$ values were 9.5 and $13.7 \mu \mathrm{M}$, respectively. These agents are known to block high-threshold noninactivating (L-type) calcium channels. For each point, $n \geq 10$. Error bars are \pm 1 binomial SD.

calcium exchanger becomes significant at higher concentrations, and opposes the calcium channel blocking effect.

\section{Tetrodotoxin does not block axonal degeneration}

Calcium can enter cells through sodium channels, which are not perfectly ion specific. The high concentration of sodium channels in axons may thus lead to significant calcium entry through these channels. In addition, a significant sodium influx could promote calcium entry via the sodium-calcium exchanger. The sodium-calcium exchanger normally works to extrude intracellular calcium by exchanging it for extracellular sodium, which can be in turn extruded by the sodium-potassium ATPase. When intracellular sodium concentrations are high, the gradient for calcium entry may exceed the gradient for sodium entry, causing the sodium-calcium exchanger to work in reverse, allowing calcium entry and extruding intracellular sodium (Stys et al., 1991; Waxman et al., 1991). Thus, a high sodium influx could result in an accumulation of intra-axonal calcium. We tested this possibility by blocking sodium channels with tetrodotoxin. There is no measurable delay in axonal degeneration after axotomy (Fig. 6) in the presence of tetrodotoxin at concentrations up to $10 \mu \mathrm{M}$.

\section{Activation of calpains during axonal degeneration}

Activation of calpains is an attractive mechanism by which an increase in intra-axonal calcium could lead to axonal degeneration (Schlaepfer and Bunge, 1973; Schlaepfer, 1974; Schlaepfer and Micko, 1978; Schlaepfer and Hasler, 1979; Schlaepfer and Zimmerman, 1984; Schlaepfer et al., 1985; Xie and Barrett, 1991). We tested the ability of two classes of calpain inhibitors to prevent axonal degeneration, the leupeptin analogs and oxiranes. Calpain Inhibitor I (N-Ac-Leu-Leu-norleucinal) and Calpain Inhibitor II (N-Ac-Leu-Leu-normethioninal) are cell-permeant leupeptin analogs. Aloxistatin is a cell permeant oxirane calpain inhibitor. All three agents preserve axotomized axons in a concentration-dependent manner, as is shown in Figure 7. 

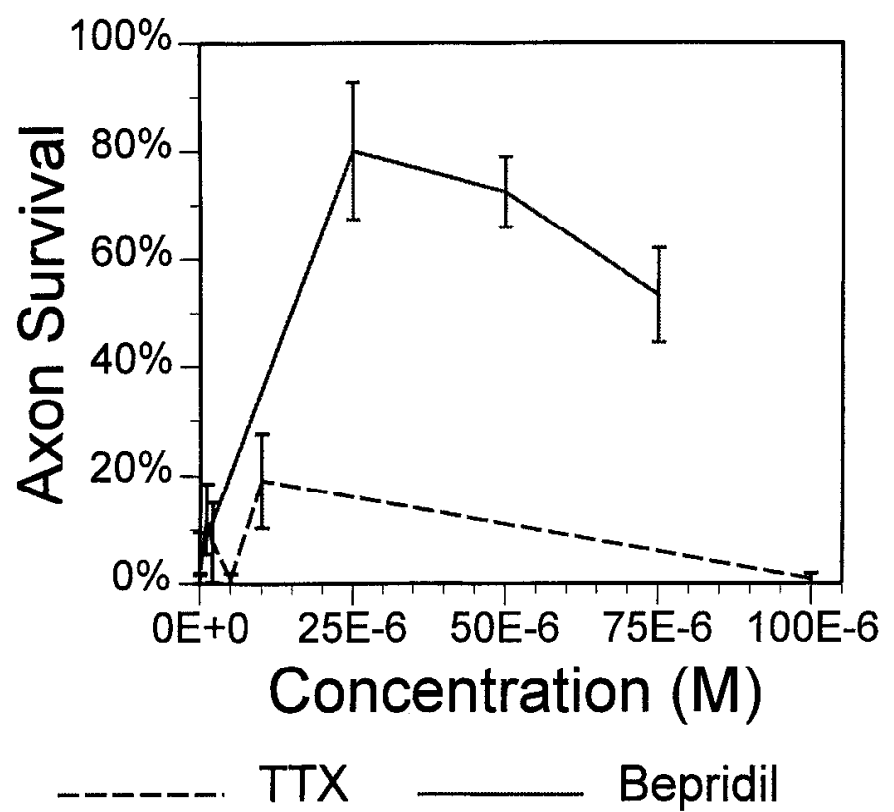

Figure 6. Tetrodotoxin (broken line) does not produce a measurable delay in axonal degeneration after axotomy. Bepridil (solid line) prevents axonal degeneration at low concentrations, but the inhibition of axonal degeneration gradually decreases with increasing concentration. For each point, $n \geq 6$. Error bars are \pm 1 binomial SD.

\section{Integrity of preserved axons}

The axolemmal and cytoskeletal integrity of axons preserved by calcium channels inhibitors was further characterized by immunostaining. Immunostaining with antibodies directed against tubulin and neurofilament confirmed staining along the length of the preserved axons (Fig. 8), which was comparable to the staining seen in uncut control axons, indicating preservation of the axonal cytoskeleton. Neuronal ubiquitin C-terminal hydrolase is a cytosolic enzyme detected by the antibody PGP9.5. Preserved axons immunostained with PGP9.5 show levels of staining (Fig. 8) comparable to controls. This observation suggests that the axolemma is preserved in the surviving fibers, and argues against the possibility that the cytoskeletal elements might be preserved in the absence of an intact axolemma.

\section{Discussion}

Our results suggest that axotomy-induced axonal degeneration involves the following sequence of events: (1) a lag-period after axotomy prior to the onset of axonal degeneration, (2) entry of calcium into the axon through an intact axolemma via a calciumspecific ion transport mechanism, (3) activation of calcium-dependent effector molecules such as calpains, (4) degradation of the axonal cytoskeleton. The later steps are potentially common to all axonal degeneration, but other triggers of axonal degeneration may involve alternate entry points into this final common pathway. While our observations are made on cultured neurons, the observed lag-period and the subsequent time course of axonal degeneration are quite consistent with observations of axonal degeneration in vivo (Chaudhry and Cornblath, 1992; Chaudhry et al., 1992). The morphology of the degenerating cultured axons is also identical to in vivo axonal degeneration, as is consistent with the fact that these primary explant cultures produce neurites whose composition and fine structure are quite representative of axons in vivo (Yamada et al., 1971; Bray et al.,

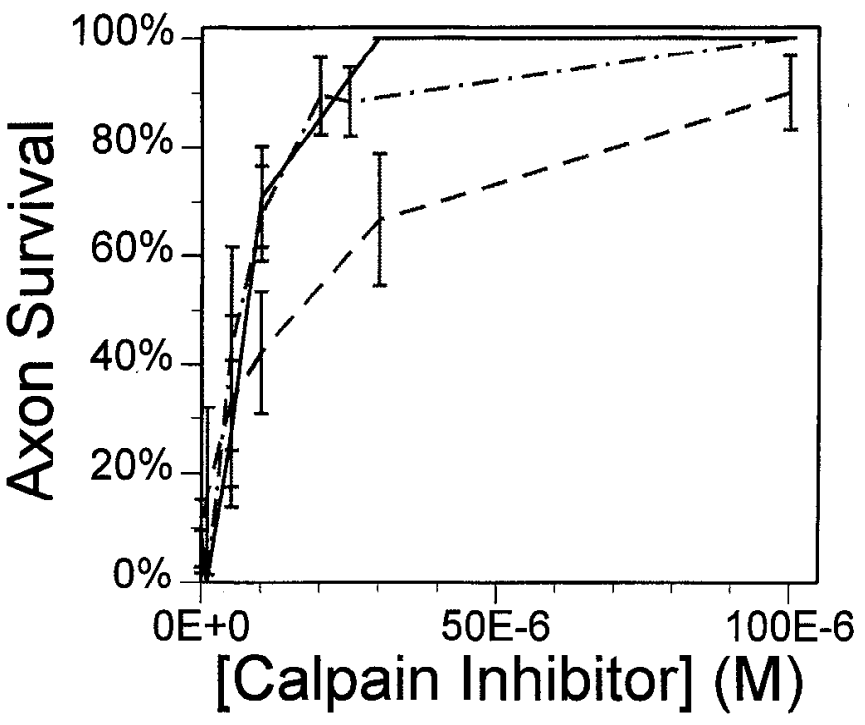

_ Calpain Inhibitor I

- _ - Calpain Inhibitor II

....- EST

Figure 7. Calpain inhibitors prevent axonal degeneration. Axonal preservation at $4 \mathrm{~d}$ is plotted against Calpain Inhibitor I concentration (solid line), Calpain Inhibitor II concentration (broken line) and Aloxistatin (EST) concentration (doubly broken line). For each point, $n \geq$ 8 . Error bars are \pm 1 binomial SD.

1978; Bray and Bunge, 1981). We suggest that the sequence of events observed in these degenerating cultured axons is a model for axonal degeneration in vivo.

\section{The lag-period}

In our cultures, there is no evidence of axonal degeneration in most axons for 12-24 hr after axotomy. This is consistent with the established ability of severed axons to quickly reseal and maintain internal homeostasis (Xie and Barrett, 1991; Ziv and Spira, 1993), and also consistent with the time course in vivo. This delay can vary between species, being typically $24-48 \mathrm{hr}$ in rats and mice but as long as $7 \mathrm{~d}$ in humans, and also seems to vary with the length of axon distal to the transection (Chaudhry and Cornblath, 1992; Chaudhry et al., 1992). Some event during this period triggers the subsequent accumulation of intraaxonal calcium, and it seems likely that this event is tied to axonal transport (Lubinska, 1977). This event may fail to occur in the Ola mutant, since increased axonal calcium produces degeneration of cultured Ola axons and calpain activity can be demonstrated in Ola axons (Glass et al., 1994).

\section{Entry of calcium into the axon}

Schlaepfer et al. (Schlaepfer and Bunge, 1973; Schlaepfer, 1974; Schlaepfer and Micko, 1978) demonstrated previously that a rise in intra-axonal calcium is associated with axonal degeneration. Our data show clearly that entry of extra-axonal calcium is a necessary component, as axonal degeneration did not proceed if the extra-axonal calcium concentration was below $200 \mu \mathrm{M}$. This is considerably greater than the normal cytosolic calcium concentration, and maintenance of the extra-axonal calcium concen- 

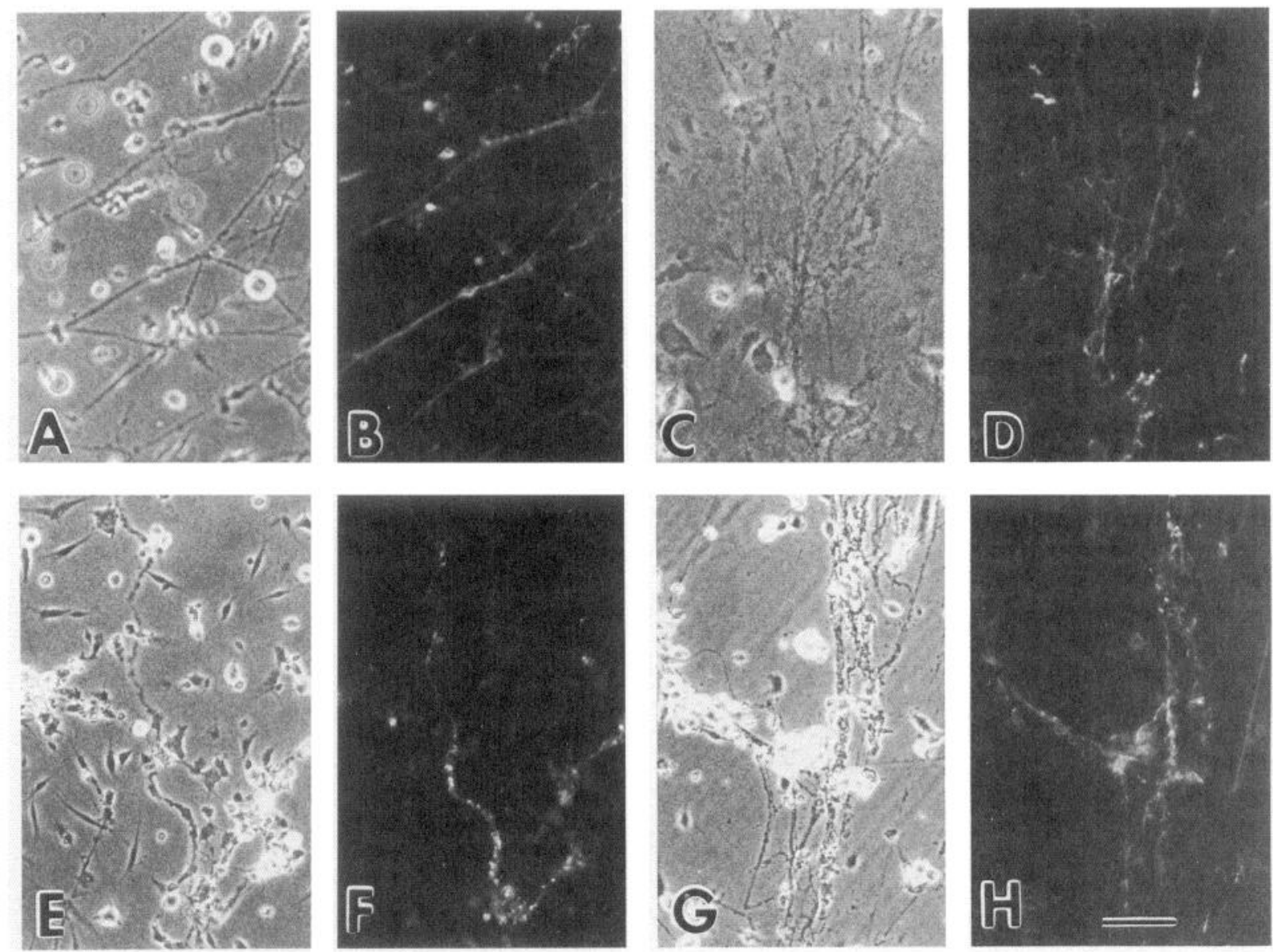

Figure 8. The axolemmal and cytoskeletal integrity of axons preserved by calcium inhibitors characterized by fluorescence immunostaining of preserved axons fixed $4 \mathrm{~d}$ after axotomy. $A$, Axons preserved in low $\left[\mathrm{Ca}^{2+}\right]$ seen by phase-contrast. $B$, The same field as $A$, immunofluorescence staining with anti-PGP9.5 antibody. $C$, Axons preserved with Calpain Inhibitor II seen by phase-contrast. $D$, The same field as $C$, immunofluorescence staining with anti-neurofilament antibody. $E$, An axon preserved in low $\left[\mathrm{Ca}^{2+}\right]$ seen by phase-contrast. $F$, The same field as $E$, immunofluorescence staining with anti- $\beta$ tubulin antibody. $G$, Axons preserved with nifedipine seen by phase-contrast. $H$, The same field as $G$, immunofluorescence staining with anti- $\beta$ tubulin antibody. Scale bar, $25 \mu \mathrm{m}$.

tration in this range did not require the presence of a calcium chelator, making it unlikely that depletion of intra-axonal calcium stores played a role in the inhibition of axonal degeneration. It remains possible that additional calcium is released from axonal stores subsequent to the entry of the extra-axonal calcium. Nonetheless, it is important to note that the requirement for an extra-axonal calcium concentration $\geq 200 \mu \mathrm{M}$ correlates well with the reported activation of m-calpain at calcium concentrations above $200 \mu \mathrm{M}$ (Mehdi, 1991).

The inhibition of axonal degeneration by cobalt and manganese, metal ions which can substitute for calcium in many processes, but which do not pass through calcium-conductive ion channels, strongly implies that calcium entry occurs through a calcium-specific ion transport mechanism. Certainly these ions should not be able to prevent calcium entry if a significant disruption of membrane integrity were the calcium entry mechanism. The lower $\mathrm{EC}_{50}$ seen with cobalt as compared to manganese is consistent with the specificity seen for binding of these ions to calcium-specific channels. It is unlikely that the inhibition of axotomy-induced axonal degeneration is due to competition with calcium at an intra-axonal site: these ions do not interfere with calcium activation of calpains, and in fact manganese can also activate calpains (Zimmerman and Schlaepfer,
1982; Mehdi, 1991). Moreover, when the ionophore A23187 is added to cultures in the presence of both inhibitory concentrations of cobalt and normal levels of extra-axonal calcium, rapid axonal degeneration occurs, confirming that these ions do not interfere with the intra-axonal mechanisms activated by calcium entry.

The effectiveness of dihydropyridines in preventing axonal degeneration also points to the involvement of an ion-specific channel. Dihydropyridines are known inhibitors of L-type calcium channels, although at high concentrations they may interfere with other types of calcium channels. L-Type calcium channels have been demonstrated on dorsal root ganglion cell bodies (Nowycky et al., 1985; Fox et al., 1987; Plummer et al., 1989; Kostyuk, 1992), but were not thought to be present in the axons. Recent evidence in other species, however, suggests that axonal calcium channels may be common, and in fact may be L-type channels (DiPolo et al., 1983; Lipscombe et al., 1988; Ziv and Spira, 1993).

L-Type calcium channels are voltage-gated channels, but the role of depolarization in the calcium entry during axotomy-induced axonal degeneration has not yet been investigated. Previous studies have demonstrated that there is a transient depolarization and increase in intra-axonal calcium immediately after 
transection, which quickly normalize once the axolemma reseals (Xie and Barrett, 1991; Ziv and Spira, 1993). The subsequent calcium entry associated with axonal degeneration does not occur until at least $24 \mathrm{hr}$ latcr. The transient changes seen immediately after transection are similar in both the axonal stump, which will not degenerate, and the isolated segment, which will go on to degenerate.

Since axonal degeneration requires extra-axonal calcium levels $\geq 200 \mu \mathrm{M}$, if calcium entry is driven solely by the concentration gradient, intracellular calcium concentrations of $\geq 200$ $\mu \mathrm{M}$ would be needed to produce axonal degeneration. If a normal resting potential of $-60 \mathrm{mV}$ were present, then an extracellular concentration of $\geq 200 \mu \mathrm{M}$ would equilibrate with an intracellular calcium concentration of $\geq 2 \mathrm{mM}$. As noted previously, a requirement for a calcium concentration $\geq 200 \mu \mathrm{M}$ correlates well with the reported activation of $m$-calpain at calcium concentrations above $200 \mu \mathrm{M}$ (Mehdi, 1991), while a requirement for $\geq 2 \mathrm{~mm}$ calcium does not appear consistent with the permeabilization data. This suggests that calcium equilibrates across a depolarized membrane, as is consistent with the in volvement of a voltage-gated channels.

The failure of $\omega$-conotoxin GVIA to inhibit axonal degeneration suggests that $\mathrm{N}$-type calcium channels are not required. $\mathrm{N}$-Type calcium channels have also been demonstrated on dorsal root ganglion cell bodies (Nowycky et al., 1985; Fox et al., 1987; Kasai et al., 1987; Olivera et al., 1987), and are thought to traverse the axon and participate in presynaptic vesicle release. T-type calcium channels have also been demonstrated on dorsal root ganglion cell bodies (Nowycky et al., 1985; Fox et al., 1987; Plummer et al., 1989; Kostyuk, 1992), and the involvement of ligand-gated calcium channels is also a possibility. None of these other channels are sensitive to micromolar concentrations of dihydropyridines, as is the L-type channel.

A further possibility is the indirect involvement of sodium channels. Waxman and coworkers have shown that axonal degeneration in central nervous system axons after ischemia involves calcium entry via the sodium-calcium exchanger (Stys et al., 1991; Waxman et al., 1991). These axons become sodiumloaded during the prolonged depolarization associated with ischemia. The lack of a sodium concentration gradient across the membrane, in the presence of a calcium concentration gradient, causes the sodium-calcium exchanger to be driven in reverse, resulting in calcium entry. This process is inhibited in ischemic optic axons by both tetrodotoxin (TTX), which prevents sodium loading, and bepridil, which can inhibit the sodium-calcium exchanger.

Bepridil is inhibitory at low concentrations in our system. Unfortunately, bepridil, like the amiloride derivatives, is a potent nonspecific calcium channel inhibitor (Kleyman and Cragoe, 1988; Braun et al., 1992; Gill et al., 1992) as well as an inhibitor of the sodium-calcium exchanger. This renders these compounds ineffective for discriminating between the "calcium channel" hypothesis and the "reversed sodium-calcium exchanger" hypothesis. Bepridil becomes less effective with increasing concentration in our system, which may reflect its ability to inhibit both calcium channels and the sodium-calcium exchanger. Thus, at low doses blockade of calcium channels may be the dominant effect, while at higher doses the inhibition of the sodium-calcium exchanger, which is normally important for calcium extrusion, may contribute to a rise in intra-axonal calcium concentrations.

TTX is ineffective as an inhibitor of axonal degeneration in our system. This may indicate that sodium loading is not involved in axonal degeneration after axotomy. TTX-resistant sodium channels are found on immature dorsal root ganglion cells, howcver, making the inefficacy of TTX inconclusive. TTX-resistant sodium channels are known to be blocked by cobalt, manganese, and phenylalkylamines (Baker et al., 1973; Kostyuk et al., 1981), but are not known to be sensitive to dihydropyridines.

\section{Activation of calpains}

Once calcium enters the axon, it is thought to activate calpains (Schlaepfer and Bunge, 1973; Schlaepfer, 1974; Schlaepfer and Micko, 1978; Schlaepfer and Hasler, 1979; Schlaepfer and Zimmerman, 1984; Schlaepfer et al., 1985), which degrade the axonal cytoskeleton, although it is possible that other calcium-dependent processes are also activated. Both the membrane permeant leupeptin analogs, calpain inhibitors I and II, and the oxirane, aloxistatin, were effective inhibitors of axonal degeneration in our system. Since the leupeptin analogs are structurally dissimilar from the oxiranes, it seems probable that their common mechanism is inhibition of calpains. In addition, as noted above, the calcium dependence of axonal degeneration in our system is similar to the calcium dependence of m-calpain. Although other calcium-activated events may occur, it appears that inhibition of calpain is sufficient to preserve the axon at the light microscope level, and prevent axonal cytoskeleton degradation as detected by immunofluorescent staining.

\section{Conclusions}

Calcium entry via an ion-specific mechanism is an early step in axonal degeneration which subsequently leads to activation of calpains. Calcium entry is both necessary and sufficient to induce axonal degeneration after axotomy, and may activate axonal degeneration under other circumstances. The activation of calpains also appears necessary and sufficient for axonal degeneration to proceed, although the role of other calcium activated processes needs to be further investigated. The mechanism by which calcium entry is initiated after a latent period of one or more days remains to he elucidated. This mechanism is probably deficient in the Ola mutant mouse, and is a potential target for therapies directed towards peripheral neuropathies.

\section{References}

Baker PF, Meves H, Ridgway EB (1973) Effects of manganese and other agents on the calcium uptake that follows depolarization of squid axons. J Physiol (Lond) 231:511-526.

Bottenstein JE, Sato GH (1979) Growth of a rat neuroblastoma cell line in serum-free supplemented medium. Proc Natl Acad Sci USA 76:514-517.

Braun S, Frey N, Herzig S, Hilbert C (1992) Potentiation of cardiopressive action among calcium antagonists from different classes: evidence for a mechanism at the single calcium channel level. Arch Pharmacol 345:586-593.

Bray D, Bunge MB (1981) Serial analysis of microtubules in cultured rat sensory axons. J Neurocytol 10:589-605.

Bray D, Thomas C, Shaw G (1978) Growth cone formation in cultures of sensory neurons. Proc Natl Acad Sci USA 75:5226-5229.

Brown MC, Perry VH, Hunt SP, Lapper SR (1994) Further studies on motor and sensory nerve regeneration in mice with delayed Wallerian degeneration. Eur J Neurosci 6:420-428.

Cancalon P (1983a) Proximodistal degeneration of C-fibers detached from their perikarya. J Cell Biol 97:6-14.

Cancalon P (1983b) Influence of temperature on slow flow in populations of regenerating axons with different elongation velocities. Brain Res 285:279-289.

Cancalon P, Elam JS (1980) Study of regeneration in the garfish olfactory nerve. J Cell Biol 84:779-794. 
Chaudhry V, Cornblath DR (1992) Wallerian degeneration in human nerves: serial electrophysiological studies. Muscle Nerve 15:687693.

Chaudhry V, Glass JD, Griffin JW (1992) Wallerian degeneration in peripheral nerve disease. In: Neurologic clinics, Vol 10, Peripheral neuropathy: new concepts and treatments (Dyck PJ, ed), pp 613-627. Philadelphia: Saunders.

DiPolo R, Caputo C, Bezanilla F (1983) Voltage-dependent calcium channel in the squid axon. Proc Natl Acad Sci USA 80:1743-1745.

Fox AP, Nowycky MC, Tsien RW (1987) Single-channel recordings of three types of calcium channels in chick sensory neurones. J Physiol (Lond) 394:173-200.

Gill A, Flaim S, Damiano B, Sit S, Brannan M (1992) Pharmacology of bepridil. Am J Cardiol 69:11D-16D.

Glass JD, Griffin JW (1991) Neurofilament redistribution in transected nerves: evidence for bidirectional transport of neurofilaments. J Neurosci 11:3146-3154.

Glass JD, Schryer BL, Griffin JW (1994) Calcium-mediated degeneration of the axonal cytoskeleton in the Ola mouse. J Neurochem 62 : 2472-2475.

Hess P (1990) Calcium channels in vertebrate cells. [Review]. Annu Rev Neurosci 13:337-356

Kasai H, Aosaki T, Fukuda J (1987) Presynaptic Ca-antagonist omegaconotoxin irreversibly blocks N-type Ca-channels in chick sensory neurons. Neurosci Res 4:228-235.

Kleyman T, Cragoe E Jr (1988) Amiloride and its anaologs as tools in the study of ion transport. J Membr Biol 105:1-21.

Kostyuk PG, Veselovsky NS, Tsyndrenko AY (1981) Ionic currents in the somatic membrane of rat dorsal root ganglion neurons. I. Sodium currents. Neuroscience 6:2423-2430.

Kostyuk PG (1992) Calcium ions in nerve cell function. Oxford: Oxford UP.

Lipscombe D, Madison D, Poenie M, Reuter H, Tsien R, Tsien R (1988) Spatial distribution of calcium channels and cytosolic calcium transients in growth cones and cell bodies of sympathetic neurons. Proc Natl Acad Sci USA 85:2398-2402.

Lubinska L (1977) Early course of Wallerian degeneration in myelinated fibers of the rat phrenic nerve. Brain Res 130:47-63.

Lunn ER, Perry VH, Brown MC, Rosen H, Gordon S (1989) Absence of Wallerian degeneration does not hinder regeneration in peripheral nerve. Eur J Neurosci 1:27-33.

Mehdi S (1991) Cell-penetrating inhibitors of calpain. Trends Biochem Sci 16:150-153.

Nowycky MC, fox AP, Isien RW (1985) I'hree types of neuronal calcium channel with different calcium agonist sensitivity. Nature 316:440-443.

Olivera BM, Cruz LJ, de Santos V, LeCheminant GW, Griffin D, Zeikus R, McIntosh JM, Galyean R, Varga J, Gray WR (1987) Neuronal calcium channel antagonists. Discrimination between calcium chan- nel subtypes using omega-conotoxin from Conus magus venom. Biochemistry 26:2086 2090 .

Plummer MR, Logothetis DE, Hess P (1989) Elementary properties and pharmacological sensitivities of calcium channels in mammalian peripheral neurons. Neuron 2:1453-1463.

Schlaepfer WW (1974) Calcium-induced degeneration of axoplasm in isolated segments of rat phrenic nerve. Brain Res 69:203-215.

Schlaepfer WW, Bunge RP (1973) The effects of calcium ion concentration on the degradation of amputated axons in tissue culture. J Cell Biol 59:456-470.

Schlaepfer WW, Hasler MB (1979) Characterization of the calciuminduced disruption of neurofilaments in rat peripheral nerves. Brain Res 168:299-309.

Schlaepfer WW, Micko S (1978) Chemical and structural changes of neurofilaments in transected rat sciatic nerve. J Cell Biol 78:369378.

Schlaepfer WW, Zimmerman UP (1984) Calcium-activated protease and the regulation of the axonal cytoskeleton. In: Axonal transport in ncuronal growth and regencration (Elam JS, Cancalon P, eds), pp 261-273. New York: Plenum.

Schlaepfer WW, Lee C, Lee VM-Y, Zimmerman U-JP (1985) An imInunublot study of neurofilament degradation in situ and during calcium-activated proteolysis. J Neurochem 44:502-509.

Stys PK, Waxman SG, Ransom BR (1991) $\mathrm{Na}^{+}-\mathrm{Ca}^{2+}$ exchanger mediates $\mathrm{Ca}^{2+}$ influx during anoxia in mammalian central nervous system white matter. Ann Neurol 30:375-380.

Tsao JW, Brown MC, Carden MJ, McLean WG, Perry VH (1994) Loss of the compound action potential: an electrophysiological, biochemical and morphological study of early events in axonal degeneration in the C57BL/Ola mouse. Eur J Neurosci 6:516-524.

Waller A (1850) Experiments on the section of glossopharyngeal and hypoglossal nerves of the frog and observations of the alternatives produced thereby in the structure of their primitive fibers. Philos Trans R Soc Lond [Biol] 140:423.

Waxman SG, Ransom BR, Stys PK (1991) Non-synaptic mechanisms of $\mathrm{Ca}^{2+}$-mediated injury in CNS white matter. Trends Neurol Sci 14: $461-468$.

Xie X, Barrett JN (1991) Membrane resealing in cultured rat septal neurons after neurite transection: evidence for enhancement by $\mathrm{Ca}^{21}$ triggered protease activity and cytoskeletal disassembly. J Neurosci 11:3257-3267.

Yamada KM, Spooner BS, Wessells NK (1971) Ultrastructure and function of growth cones and axons of cultured nerve cells. J Cell Biol 49:614-635.

Zimmerman U-JP, Schlaepfer WW (1982) Characterization of a brain calcium-activated protease that degrades neurofilament proteins. Biochemistry 21:3977-3983.

Ziv N, Spira M (1993) Spatiotemporal distribution of $\mathrm{Ca}^{2+}$ following axotomy and throughout the recovery process of cultured Aplysia neurons. Eur J Neurosci 5:657-668. 